\title{
Identificación de las representaciones de ambiente y educación ambiental que circulan en la escuela
}

\author{
María Rocío Pérez Mesa* \\ Yair Alexánder Porras ${ }^{* *}$ \\ Raúl Alfonso González ${ }^{* * *}$
}

Artículo recibido: 20-2-2006 y aprobado: 2-5-2007

Identification of the environment and environmental education representations that go around in the school

Resumen: El propósito del presente artículo es identificar el tipo de representaciones sociales que manifiestan docentes y estudiantes de instituciones educativas de Bogotá, en relación con el ambiente y la educación ambiental, por ser éstas un cuerpo de conocimientos que permiten a los individuos establecer un sistema de valores, actitudes e ideas, que hacen inteligible la realidad ambiental y favorecen el establecimiento de vínculos a nivel colectivo, que se traducen en determinados tipos de prácticas sociales.

Palabras clave: representaciones sociales, ambiente, educación ambiental, proyectos ambientales escolares.
Abstract: This article aims at identifying the kind of social representations that teachers and students who belong to public educational institutions have in relation to environment and environmental education, because they constitute a knowledge field that allows the individuals to build a system of values, atitudes and ideas, that make the environmental reality intelligible and work in favour of the links establishment that is required for making possible certain sorts of social practices.

Key words: social representations, environment, environmental education, environmental projects.

* Profesora de la Universidad Pedagógica Nacional. Departamento de Biología. Coordinadora del grupo de investigación La Educación Ambiental en el Contexto Educativo Colombiano.

** Profesor de la Universidad Pedagógica Nacional. Departamento de Biología. Coinvestigador del grupo de investigación La Educación Ambiental en el Contexto Educativo Colombiano.

*** Coinvestigador del grupo de investigación La Educación Ambiental en el Contexto Educativo Colombiano.

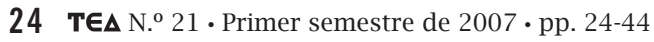




\section{Introducción}

Somos constructores de significados que compartimos socialmente (Pozo, 1998), permanentemente utilizamos las creencias, teorías y explicaciones que elaboramos sobre el mundo para comprender la realidad, reducir la incertidumbre frente a los problemas y adelantar acciones que materialicen nuestros pensamientos. Una idea puede transformar el estado de cosas, así un conjunto de representaciones que motivan el cambio en nuestra manera de sentir, pensar y actuar, y que además despiertan un creciente interés debido al tipo de repercusiones sociales, económicas, políticas, culturales y educativas que concitan, es sin duda el ambiente y la educación ambiental.

En el caso particular de las representaciones de ambiente y de educación ambiental que utilizan docentes y estudiantes en el contexto educativo, resulta pertinente analizar algunos de sus elementos que pueden ser significativos y diversos a la hora de pensar, planear, comunicar ideas y actuar a partir de una posición ideológica. El aprendizaje vivenciado y las representaciones que se tienen en una comunidad educativa en relación con la dinámica biofísica, social y cultural, se exponen en el presente estudio, con el ánimo de confrontar nociones, construir conceptos y elaborar argumentos frente al significado y sentido que revela la dimensión ambiental en la práctica educativa, la cual se articula a una visión de hombre, educación, sociedad y cultura.

La consolidación de una identidad ambiental en cada institución, parte de la reflexión sobre el tipo de estrategias pedagógicas, didácticas y metodológicas que propician la interacción entre los procesos educativos involucrados en la construcción de la cultura colombiana y el papel que desempeña la formación ambiental en el desarrollo de un ideario de ciudadanía, pues como señala Sanmartí y Pujol (2002) la acción asociada a la educación ambiental se introdujo anteponiendo el aprendizaje de contenidos, debido a la necesidad de sensibilizar y dar información. Esta rutina, que aún se mantiene en algunas instituciones, ha venido transformándose en términos de toma de decisiones y capacitación para la acción, lo cual favorece un entendimiento de los problemas locales, regionales y globales a partir de los esfuerzos, en ocasiones aislados, de docentes y estudiantes.

\section{Problema}

Para comprender la complejidad de las representaciones que elaboran los sujetos cuando interactúan con su medio, es necesario asumir una mirada crítica, integradora y dialéctica, que puede ser interpretada desde la educación ambiental, la cual permite pensar el ambiente más allá de una realidad dada y objetiva, manipulable científica y técnicamente, para considerarla como una construcción intersubjetiva y crítica, en donde circulan saberes y emergen redes de interacciones entre los sujetos. Relacionar el contexto histórico, político y ético en el que son elaboradas dichas representaciones, permite entender el ambiente como una construcción social y cultural, orientada hacia la transformación de realidades.

El fortalecimiento de espacios de discusión en donde se ausculten las representaciones sociales sobre ambiente, propicia el reconocimiento de la 
diversidad biológica, social y cultural de los ciudadanos, invita a exaltar la responsabilidad social de los docentes, legitimando su papel como dinamizadores de la cultura, al diseñar estrategias que favorecen el respeto por las minorías, la comprensión de los procesos históricos y sociales, el análisis de la evolución de los conocimientos, las expresiones artísticas y estéticas, la comunicación en diferentes contextos y, a su vez, como miembros de una aldea global, el desarrollo de una ética ambiental que se base en la equidad y la complementariedad, dentro de un proyecto político de sociedad. En este sentido se formula como pregunta de investigación: ¿Qué tipo de representaciones sobre ambiente y educación ambiental circulan en instituciones educativas de Bogotá?

\section{Marco de referencia}

\section{Concepto de ambiente}

Reconocer las comunidades como sistemas complejos que evolucionan identificando sus interacciones comunes, favorece la participación de sus miembros en las decisiones filosóficas, políticas, sociales, éticas, estéticas, jurídicas y científicas que les competen. Esto posibilita de esta manera la construcción de procesos dialógicos en los que se concilia el estudio de problemas comunes que contribuyen al desarrollo de habilidades, valores y actitudes, indispensables en el desarrollo de estrategias tendientes al mejoramiento de la calidad de vida de los grupos sociales.

El ambiente se percibe como un constructo social, mediado por elementos culturales y representaciones simbólicas, que están ideológica y políticamente condicionadas y que ejercen un papel decisivo en los modos de interpretar la vida cotidiana, de construir valores, de desarrollar conocimientos, de hacer una lectura y comprensión crítica del entorno, como base para la construcción de un mundo democrático y justo.

Resulta conveniente resaltar el hecho de que no existe una única representación de ambiente, que si bien podemos encontrar una multiplicidad de visiones teóricas frente a su conceptualización, que lejos de ser un inconveniente evidencian la complejidad y riqueza del mismo, nos permiten examinar los micromundos ${ }^{1}$, los contextos de significación de los sujetos y las interacciones propias de las dinámicas educativas, sociales y culturales de las comunidades, enmarcadas en una visión pluriparadigmática de los acontecimientos (Pérez y Porras, 2005).

De acuerdo con Talero y Umaña (1993), el ambiente debe concebirse como un sistema complejo, global y dinámico, conformado básicamente por tres grandes subsistemas que tienen su propia dinámica y que interactúan entre sí con mayor o menor intensidad y complejidad en forma permanente: el subsistema físico-natural, denominado también ambiente natural o biofísico; el subsistema sociocultural, denominado también ambiente social, y un subsistema creado, particular, en el cual se desenvuelve el ser humano manteniendo estilos de vida, culturas y tradiciones propias de su grupo en donde adopta formas de satisfacer sus necesidades básicas.

\footnotetext{
Según Varela (1996) un micromundo es "un espacio donde un ser humano se constituye como tal en un sistema social donde hay una recurrencia en una interacción de cooperación y se establece una autoconciencia".
} 


\section{Representación social}

Existen sistemas de ideas particulares que utilizan las personas para interpretar, entender la realidad y vincularse al grupo social. Este cuerpo de conocimientos que se asocian con las teorías implícitas o de sentido común, hacen parte de una forma de pensamiento social (Jodelet, 1986) que permite a los sujetos entender el mundo material e iniciar una actividad representativa, por lo que su examen resulta particularmente importante, debido al grado de significación que implica para el presente estudio. Este sistema de conocimientos se denomina representación social, que para el caso del ambiente y la educación ambiental constituye una primera mirada al sistema de valores, ideas y prácticas que manejan docentes y estudiantes. Estas teorías, que trascienden las imágenes y las actitudes, propician el establecimiento de una organización que permite a los individuos orientarse en el mundo material y social. Además, posibilitan la comunicación entre los miembros de una comunidad a través del establecimiento de un intercambio social a partir de una realidad común que se alimenta de la historia individual y grupal.

La representación social es una modalidad particular del conocimiento, cuya función es la elaboración de los comportamientos y la comunicación entre los individuos. La representación es un corpus organizado de conocimientos y una de las actividades psíquicas gracias a las cuales los hombres hacen inteligible la realidad física y social, se integran en un grupo o en una relación cotidiana de intercambios, liberan los poderes de su imaginación (Moscovici, 1979) .

\section{Representaciones de ambiente} y educación ambiental

De acuerdo con Reigota (1995), no existe un consenso general sobre el concepto de representación social en educación ambiental, pero su estudio debe ser la base de la búsqueda de negociación y solución de los problemas ambientales, pues "no se trata de saber cuantitativamente más, sino cualitativamente mejor sobre las cuestiones que un grupo determinado pretende estudiar y donde pretende actuar". Para este autor existen tres tipos de representaciones sociales sobre ambiente y educación ambiental, las cuales se categorizan en: naturalista, globalizadora y antropocéntrica. La representación social naturalista evidencia aspectos naturales, enfatizando sobre el estudio de factores biofísicos que se abordan desde la ecología; la globalizadora se caracteriza por resaltar las interacciones entre los aspectos sociales y naturales, y la antropocéntrica presenta la utilidad de los recursos naturales como garantía de la sobrevivencia de los seres humanos.

Por otra parte, Sauvé (1994) considera que las representaciones de ambiente que una persona o un grupo adopten, necesariamente determinan sus comportamientos, actitudes y acciones. El análisis de este sistema de ideas constituye un paso previo a la implementación de cualquier tipo de solución externa - que usualmente es de corte técnico-científico- a las problemáticas de las comunidades. Las principales representaciones de ambiente, propuestas por Sauvé, que evidencian el tipo de interacción que los individuos de un grupo social establecen entre sí, son: 
El ambiente como problema por resolver: esta primera visión se centra en el estudio del subsistema biofísico, con aquello que está amenazado por la polución, por las molestias y por los deterioros. Se debe aprender a preservar y restaurar la naturaleza. Como estrategias pedagógicas encontramos aquellas que contribuyen al desarrollo de habilidades, relativas a la resolución de problemas.

El ambiente como recurso para administrar: esta visión de ambiente se refiere al patrimonio biológico colectivo, asociado a la calidad de vida. Sin embargo, se trata de un recurso limitado que se agota y se degrada. La educación para el desarrollo sostenible se ha convertido en uno de los marcos de referencia, al inscribirse en la perspectiva del ambiente como recurso centrando su interés en "promover la utilización creativa y efectiva del potencial humano y de todas las formas de capital para asegurar un crecimiento económico rápido y más equitativo que reduzca el impacto ambiental" (Unesco, 1993, citado por Sauve, 1994). Sin embargo, la autora plantea una mirada crítica a esta tendencia pues promueve la competitividad y la productividad, cuyo propósito es una educación con una visión sociocultural de corte industrial.

El ambiente como naturaleza para apreciar, respetar y preservar. es el ambiente original, puro, del cual el hombre se ha disociado y con el cual debe aprender a renovar los vínculos con el fin de enriquecer su calidad de ser, para algunos se trata ante todo de una naturaleza prístina que debe ser admirada y respetada. La estrategia pedagógica planteada busca desarrollar una sensibilidad hacia la naturaleza y un conocimiento de su realidad a través de nuestros sentidos. Busca también que tomemos conciencia que formamos parte de ella.

El ambiente como biosfera para vivir juntos por mucho tiempo: el ambiente corresponde a un mundo acabado, a un organismo autorregulador, lo cual se deriva de los planteamientos de Jacquart, Morin, Lovelock (citados por Sauvé, 1994). Es el objeto de la conciencia planetaria cósmica (Gaia). Como estrategia pedagógica se destaca la de confrontar al sujeto con una situación problemática que implica una dimensión moral. Este modelo de pensamiento sociocientífico busca que los estudiantes sean capaces de tomar decisiones claras, eficaces y responsables en el mundo actual y futuro, dominado por la ciencia y la tecnología, caracterizado por la rapidez de los cambios y por la degradación del ambiente.

El ambiente como medio de vida para conocer, para administrar: el ambiente cotidiano representado en la escuela, la casa, el trabajo y en el ocio. Este medio de vida está impregnado de elementos humanos socioculturales, tecnológicos, históricos, etc. Es el ambiente propio ante el cual cada quien debe desarrollar un sentimiento de pertenencia. En relación con la educación, la estrategia pedagógica debe asociar la educación ambiental con la vida cotidiana, para el desarrollo de una teoría contextual de la cotidianidad. De lo que se trata es que todos seamos creadores y constructores de nuestro propio medio de vida (el contexto como fuente y objeto del conocimiento).

El ambiente comunitario para participar: el ambiente es una colectividad humana, un medio de vida compartido 
con sus elementos naturales y antropológicos. Es el lugar de la solidaridad, de la vida democrática, en el que se debe aprender a implicarse para participar en su evolución. El modelo pedagógico es la investigación-acción para la resolución de problemas comunitarios, es el propuesto o sugerido. Aquí se busca que los estudiantes, desde sus propios contextos, investiguen su realidad y puedan identificar problemas ambientales para resolverlos a partir de la cooperación.

Dado que el ambiente es un sistema complejo, la complementariedad de los enfoques y de las estrategias educativas favorecerán una mejor comprensión que permitan resolver la diversidad de problemáticas ambientales.

Las diferentes visiones sobre el ambiente y las diversas prácticas educativas relativas se complementan unas a otras en la educación relativa al ambiente. El ambiente es una realidad infinitamente compleja y contextual, que sólo se puede abordar en forma adecuada desde una pluralidad de perspectivas. El ambiente es a la vez recurso, biosfera, medio de vida, proyecto comunitario, problema, posibilidad (Sauve, 1994).

\section{Los Proyectos Ambientales Escolares (Prae)}

Dentro de los referentes conceptuales que apoyan la presente investigación, se destacan los planteamientos de Torres (1996) en torno a los Proyectos Ambientales Escolares (Prae), considerándolos como aquellos

que desde el aula de clase y desde la institución escolar se vinculan a la solución de la problemática ambiental particular de una localidad o región, permitiendo la generación de espacios comunes de reflexión, desarrollando criterios de solidaridad, tolerancia, búsqueda de consenso, autonomía y preparando para la autogestión en la búsqueda de un mejoramiento de la calidad de vida, que es el propósito último de la educación ambiental.

De igual manera, la misma autora esgrime las razones por las cuales los Prae pueden incorporarse a la realidad institucional de la escuela, al plantear la posibilidad de que éstos respondan desde un tema o un problema del Proyecto Educativo Institucional (PEI) pero sin olvidar lo fundamental: "que sean interdisciplinarios y busquen la integración con el ánimo de que su proyección tenga incidencia directa en la formación integral de los estudiantes y los prepare para actuar, consciente y responsablemente, en el manejo de su entorno" (Torres, 1996).

De acuerdo con el Decreto 1743 de 1994, de la Ley General de Educación Nacional de Colombia, algunos parámetros establecidos para la elaboración e implementación de un Prae son:

- Basarse en la investigación en educación ambiental y para el desarrollo sostenible, resolviendo los problemas del entorno de manera sistémica (abarca múltiples teorías del conocimiento) con un enfoque dialéctico (buscar soluciones en consenso).

- Ser un proyecto pedagógico enriquecido por el entorno que permita la organización y participación comunitaria en el ámbito local y regional.

- Tener carácter interdisciplinario, explorando los enfoques de las diferentes áreas del conocimiento con el fin de resolver problemas ambientales propios de las comunidades.

- Estar basado en la construcción de modelos pedagógicos y didácticos 
que posibiliten la aproximación al conocimiento ambiental y que permitan indagar, experimentar, probar e integrar la dimensión ambiental a todos los elementos del currículo.

- Integrar la labor docente a la solución y manejo de problemas ambientales, construyendo espacios integradores e interdisciplinarios para la reflexión y la acción permanentes.

- Ofrecer una proyección que tenga incidencia directa en la formación integral del individuo, preparándolo para actuar consciente y responsablemente en el manejo de su entorno.

- Basarse en el respeto, la tolerancia y tener en cuenta los conceptos de participación, autonomía, gestión y concertación, integrando a toda la comunidad desde la escuela.

- Estar encaminado a desarrollar conciencia, conocimientos, actitudes, aptitudes y capacidad de autoevaluación y participación permanentes.

- Tener claros referentes contextuales, naturales, sociales y culturales, lo mismo que una apropiada identificación de los actores fundamentales del proceso, de sus intereses, de sus voluntades, sus aspiraciones, necesidades y en general de sus inquietudes en torno al ambiente.

Los Prae posibilitan la integración de las diferentes áreas del conocimiento, las diversas disciplinas y los diversos saberes, para permitir a estudiantes, docentes y comunidad, la comprensión de un universo conceptual aplicado a la resolución de problemas locales, regionales y nacionales.

\section{Plano ético}

La educación ambiental contribuye a la formación del ciudadano en cuanto favo- rece el desarrollo de actitudes y valores del sujeto, siendo posible trabajar, de acuerdo con las directrices del Ministerio de Educación Nacional, los tres grandes grupos de competencias ciudadanas, a saber: convivencia y paz; participación y responsabilidad democrática; pluralidad, identidad y valoración de las diferencias. En este sentido, la competencia de convivencia y paz, entendida como la consideración de cada persona como ser humano, es desarrollada a partir de la legitimación de la dimensión social, lo que permite el fortalecimiento de valores como la empatía, el respeto y la tolerancia; la participación y responsabilidad democrática se evidencian en la toma de decisiones del individuo cuando interactúa en un determinado contexto, lo cual implica el reconocimiento de sí mismo y del otro como un ser activo dentro de la sociedad; y por último, la pluralidad, identidad y valoración de las diferencias parten del entendimiento de la diversidad humana al comprender el entramado social desde su complejidad, con el cual se construye socialmente la cultura (Novo, 2003).

En este orden de ideas, resulta evidente que la educación ambiental favorece la formación del ciudadano, en cuanto se comprende más allá del subsistema físico-natural, dando paso al subsistema sociocultural en el que participan diversos factores que permiten contribuir a la formación de personas capaces de analizar y aportar en procesos colectivos, de tomar decisiones que favorezcan el progreso de la sociedad, de convivir con los demás en un ambiente de tolerancia y respeto y, por supuesto, de escuchar al otro y aunar elementos con el fin de llegar a la formulación de una idea enriquecedora. 


\section{Plano metodológico}

Existen múltiples abordajes para el trabajo en educación ambiental. Algunos autores plantean tres tipos de estrategias ambientalizadoras del currículo: la tecnológica, la política y la cultural. La primera sugiere que la implementación del discurso tecnológico en relación con la educación ambiental ha sido de carácter instrumental, no corresponde con el contexto escolar y obedece más a una postura de tipo tecnócrata (García, 2002). La estrategia política se asume como una postura de carácter participativo que surge de las motivaciones e intereses individuales y colectivos que desembocan en compromisos producto de la cooperación y la negociación. La estrategia cultural referida a la construcción de un pensamiento ambiental caracterizado por una nueva visión de mundo que implica una reflexión profunda en torno a los sujetos, a sus relaciones, a sus costumbres, sus creencias, sus conocimientos, sus comportamientos, sus actitudes. En la escuela, esta perspectiva metodológica se puede abordar a partir de un trabajo en equipos interdisciplinarios y métodos activos en el que el eje central del acto pedagógico se enfoque en la problematización, reorientación de los contenidos y uso de la creatividad.

\section{Metodología}

El equipo investigador considera pertinente indagar en aquellas instituciones que presentan Proyectos Ambientales Escolares (Prae), teniendo como categorías de análisis aspectos como: los tópicos generadores; la conceptualización sobre ambiente; la articulación del Prae al PEI; el tipo de proyecto, metodologías y estrategias empleadas; las transformaciones evidenciadas; los objetivos y la evaluación.

Para cumplir con estos propósitos se utilizaron como instrumentos de investigación la entrevista y la encuesta, las cuales permitieron configurar diferentes elementos orientados desde el problema de investigación (Cerda, 1998). Cada instrumento se ha pensado y diseñado para recoger aspectos específicos de la muestra constituida por docentes y estudiantes de las instituciones educativas del sector oficial. El equipo diseñó y evaluó los instrumentos con antelación a la prueba piloto, con la intención de disminuir los factores de dispersión, sesgo o desviación de la intencionalidad de dichos instrumentos. A su vez se diseñó y aplicó una encuesta de pregunta abierta a estudiantes, para obtener información en el propio lenguaje de los encuestados.

\section{Población y muestra}

La población de estudio se extendió a 19 localidades de las que forman parte 392 instituciones educativas del sector oficial del Distrito Capital. Se trabajó con una muestra de 24 instituciones oficiales del sector urbano, distribuidas en 13 localidades. Los criterios de selección de estas instituciones fueron: diligenciamiento de los instrumentos suministrados, existencia de proyectos ambientales en las instituciones educativas, tópico generador del trabajo ambiental, conceptualización, articulación al PEI, tipo de proyecto, metodología, estrategias empleadas, transformaciones. 


\section{Resultados y análisis}

A continuación se presentan los resultados y análisis de la investigación a partir de la aplicación de instrumentos como entrevistas y encuestas dirigidas a estudiantes, docentes y directivos docentes que participan del trabajo ambiental en cada una de las instituciones que conforman la muestra del presente estudio.

\section{Encuesta N. ${ }^{\circ} 1$. Análisis de Proyectos Ambientales Escolares}

La sistematización de la información, proporcionada por este instrumento, fue organizada en siete categorías con relación al trabajo ambiental (Prae, proyectos de aula), que se ha venido adelantando en las diferentes instituciones educativas, como se presentan a continuación:

\section{Tópico generador}

De acuerdo con lo escrito en el documento correspondiente a los Prae de la muestra, se encuentra cierta tendencia a asumir la realidad como algo externo al sujeto, a partir de lo concreto, de lo visible, lo manipulable, centrando el problema en solucionar el manejo inadecuado de los residuos sólidos. En este sentido, se evidencia la representación antropocéntrica de la educación ambiental -de acuerdo con Reigota (1994)-, como la mejor forma de administrar los recursos para garantizar la sobrevivencia, revelando una brecha entre los modelos de educación ambiental que resultan externos al grupo social, con aquellos que legitiman una realidad construida a partir de la interacción entre los sujetos. Es de resaltar, el tratamiento que se le da en muchos casos al concepto de ambiente como lo estético en donde se advierte, así mismo, una orientación ecologicista.
Algunas instituciones analizadas destacan, dentro de sus fortalezas, la creación de un espacio en el que emergen cierto tipo de interacciones entre los sujetos, manteniendo, a su vez, una relación dinámica con su entorno. Estas instituciones, explicitan en sus documentos, el impacto que tiene la contaminación por residuos sólidos en la calidad de vida de los estudiantes, existiendo una preocupación sentida por el desarrollo humano. También es común encontrar en las instituciones analizadas, el manejo de un discurso en el cual se privilegian los conocimientos, actitudes y valores, para transformar ciertas acciones que pueden contribuir con el mejoramiento del ambiente, ratificando de esta forma los planteamientos que se generan desde entidades como el Ministerio de Educación y el Ministerio de Ambiente, Vivienda y Desarrollo Territorial.

\section{Conceptualización}

Dentro de las conceptualizaciones desarrolladas en los documentos de las instituciones sobre educación ambiental, se presentan diferentes definiciones, que dan cuenta del tipo de manejo y fundamentación teórica que se vislumbra en la dinámica institucional. En un 20\% de las instituciones se aborda la educación ambiental como un conjunto de recursos informativos y formativos, situando como parte de los referentes conceptuales, el comportamiento proambiental desde la psicología ambiental (García, 2002), que tiene por objeto adelantar acciones relacionadas con el manejo de los residuos sólidos.

La conceptualización que se hace de educación ambiental en otras instituciones de Bogotá, se asocia con un proceso 
democrático y dinámico, prevaleciendo aquella postura que reconoce al otro y considera fundamental garantizar una buena calidad de vida desde la sostenibilidad. En este sentido hay una mirada antropocéntrica, que es consistente con el tópico generador.

Otras instituciones basan su conceptualización en los documentos generados por el Ministerio de Educación en torno a la educación ambiental. La utilización de una bibliografía en la que aparecen diferentes autores, reconocidos en el trabajo ambiental, les posibilita establecer relaciones con otros aspectos como: contaminación, residuos sólidos, la ciudad y los parques. Un cuarto grupo de instituciones considera relevante relacionar la evolución biológica con la cultural, situando al ser humano como agente de cambio que propicia interacciones permanentes entre aspectos sociales y naturales, en una clara alusión a la representación globalizante de Reigota (1995), en la que se establece un manejo responsable de los recursos.

\section{Articulación al PEI}

En términos generales, los Prae se encuentran articulados al PEI de las instituciones desde los valores (sentir, pensar y expresarse en la vida, para la vida y la libertad), creando un lazo directo con los conocimientos, fomentando en los individuos la adquisición, el desarrollo de destrezas y actitudes necesarias para comprender y apreciar las interrelaciones entre el hombre, su cultura y el medio físico.

El Prae, en un porcentaje bajo de las instituciones, se encuentra articulado desde la visión y misión del PEI, resaltando la recuperación de los contextos significativos de aprendizaje, que pro- mueven el desarrollo humano, destacando la formación en valores. En pocas instituciones no se explicita, de acuerdo con la revisión de los documentos, la articulación del Prae con el PEI.

\section{Objetivos}

En términos generales los objetivos están relacionados con la sensibilización, el mejoramiento de la calidad de vida, el fortalecimiento de valores, la organización de grupos ambientales y ecológicos, al igual que la resolución de problemas prácticos relacionados con la recuperación y cuidado de los espacios físicos, correspondiéndose a su vez con la metodología y las estrategias planteadas.

Tipo de proyecto, metodología, estrategias empleadas

En un número muy reducido de instituciones, el desarrollo del Prae se asume como un proyecto de investigación de corte cualitativo fundamentado en la IAP, que se desarrolla a través de proyectos. Al emplear como metodología el uso del medio como centro de interés, de carácter vivencial y de encuentros con otros espacios menos intervenidos, se propicia un contraste con la ciudad y más directamente con la situación ambiental de la localidad, mostrando una visión ideal de lo que debe ser la naturaleza y la relación del hombre con el medio.

En otras instituciones se plantean tres fases de trabajo: una de sensibilización, otra de problematización y una tercera de experimentación. Además se presenta, como parte de los documentos, la sistematización de las experiencias realizadas en cada año de ejecución del Prae. En cuanto a las 
estrategias empleadas se mencionan: la comparación ambiente natural versus ciudad, a través de salidas de campo, videoforos, talleres, casa ecológica y la conformación de grupos ambientales (representación naturalista). Así mismo, se incluyen reuniones periódicas del grupo de maestros de ciencias naturales y de otras áreas, para acordar las actividades y la intencionalidad de las mismas con la comunidad educativa.

En pocas instituciones no se puede determinar si se hacen procesos de investigación; sin embargo, utilizan como metodología la elaboración de proyectos de intervención. Estos colegios proponen como estrategias la conformación del grupo ambiental, el diseño de rutas ambientales, las salidas ecológicas, el juego de roles y la elaboración de trabajos artísticos. En algunos se hace adicionalmente la recuperación de zonas verdes, aunque no aparece una sistematización de las experiencias realizadas.

Algunas instituciones hacen especial énfasis en las salidas de campo como estrategia de integración de las diferentes áreas, para abordar las problemáticas ambientales y así posibilitar una mirada interdisciplinar.

\section{Transformaciones}

En todas las instituciones, las transformaciones están relacionadas fundamentalmente con propuestas de gestión y de intervención, pero no se explicitan transformaciones de tipo conceptual. Igualmente se expresan cambios en las actitudes y valores de los estudiantes aunque no se fundamenta cómo es el desarrollo de dicho proceso.

Dentro de las dinámicas propias de cada institución, existen transfor- maciones comunes entre las que se encuentran: la consolidación de grupos ecológicos, que en cierta medida interactúan con la comunidad. En algunos casos particulares, los cambios se evidencian con la recuperación de zonas verdes y parques, contando con la participación de la comunidad. Estos procesos de organización tienen como objetivo, en pocas instituciones, la conformación de una cooperativa y el fondo de solidaridad para la ayuda de los estudiantes. Otras actividades se centran en la institucionalización de la casa ecológica como centro de acopio de papel, la participación de estudiantes en actividades de educación ambiental y la recuperación del espacio público.

Es coincidente encontrar en varias instituciones, la distribución de canecas como parte de la preocupación estética, la conformación de zonas verdes y un mejoramiento de las actitudes hacia el ambiente. También es común encontrar el interés por parte de los colegios de incentivar tanto el sentido de pertenencia por la institución como la autonomía.

Es de resaltar, la importancia que se le brinda a las salidas de campo como un punto coyuntural en el que se propicia la integración de las diferentes áreas, hecho que permite abordar las problemáticas ambientales de manera global y así posibilitar una mirada interdisciplinar.

\section{Análisis de las entrevistas realizadas a los docentes \\ Consideraciones en relación con las características de los proyectos ambientales escolares}

Un proyecto ambiental es considerado por los docentes, como tal, si reúne 
ciertas condiciones en términos actitudinales, axiológicos, deontológicos y si además, procura cambios individuales y colectivos. Las afirmaciones que se establecen en torno a este criterio van desde el propiciar espacios y condiciones que permitan un desarrollo óptimo para la vida, hasta la argumentación que evidencia la influencia del hombre en el desarrollo de los seres vivos en general, los otros seres humanos en particular y el medio físico; así mismo, los docentes creen fundamental desarrollar el proyecto ambiental para comprender que el ambiente es un constructo inherente a la vida, en donde los valores hacia el respeto por sus elementos constitutivos, vienen a determinar el objetivo de formación.

\section{El concepto de ambiente y educación ambiental que circula en la escuela}

Resulta significativo encontrar docentes que realizan una crítica depurada a la representación de ambiente que usualmente circula en la escuela, estimando la no existencia de dicho concepto, pues sostienen que si bien existe una definición teórica del mismo, no se explicita en la interacción de los individuos. Así mismo, afirman que se desconoce el espacio relacional de convivencia en el que se debería construir dicho concepto y no se evidencia el reconocimiento de la realidad próxima al núcleo escolar. Se destaca la reflexión de los docentes hacia una representación de ambiente que trasciende lo biofísico, integrándolo a la dinámica educativa y a la vida de los estudiantes (representación globalizante). En algunas instituciones se concibe la educación ambiental como un proceso de concientización de los estudiantes sobre la conservación y recuperación del entorno, así como de sus relaciones con los demás.

\section{La educación ambiental en el Prae. Consideraciones desde lo pedagógico y didáctico}

Desde lo pedagógico, la educación ambiental se aborda siguiendo los parámetros del Ministerio de Educación Nacional, orientando su acción hacia la participación de las diferentes áreas del conocimiento. Debido al abordaje casi exclusivo del Prae desde las ciencias naturales, se asume lo pedagógico como un proceso de formación en el que se propicia la resolución de problemáticas ambientales. Así mismo se vislumbra cierto activismo en las respuestas de los docentes, al atender algunas demandas de las instituciones en cuanto a la reducción de los residuos sólidos, el ornato y la conservación, pero careciendo de una perspectiva pedagógica profunda, al no tenerse en cuenta la reflexión permanente en torno al saber y a la práctica educativa (representación naturalista).

En cuanto a lo didáctico, se evidencia un marcado interés por el desarrollo de proyectos, a partir de la investigación, la resolución de problemas de lápiz y papel, las salidas de campo y el desarrollo de talleres que resultan una opción novedosa, frente a las temáticas planas que se desarrollan en el aula de clase. A pesar de que no se explicita una fundamentación teórica frente a lo didáctico, se asume como el hacer de los estudiantes dentro de una perspectiva diferente a la tradicional, por lo que se desconoce, en parte, su impacto como una práctica educativa cargada de ideología, que tiene en su seno la reflexión sobre la enseñanza. 
Contribuciones de la educación ambiental a la construcción de una realidad ambiental escolar

Las respuestas se centran en la motivación del estudiante por conocer una realidad que se encuentra dada, expedita, por lo que las salidas de campo, en las que se cambia el escenario propio de la ciudad (cemento, calles, contaminación) resultan claves para justificar la conservación y el mejoramiento de ese ambiente externo, prevaleciendo las acciones mecanizadas para obtener respuestas efectivas (cerrar las llaves, botar papeles en la caneca, cuidar las zonas verdes, etc.).

\section{Incorporación de la dimensión ambiental al currículo}

La mayoría de instituciones relacionan la educación ambiental con los progresos y propuestas lideradas desde el área de ciencias naturales. Aquí se incorporan de manera exclusiva las temáticas ambientales desde el punto de vista ecológico, a pesar de validar discursos integradores que propugnan el abordaje de problemas ambientales, desde una perspectiva interdisciplinar. En pocas instituciones la articulación entre la educación ambiental y el currículo se desarrolla a partir de proyectos, talleres y trabajos.

\section{Motivaciones de los docentes por el trabajo en educación ambiental}

Conciencia por mejorar el ambiente y sensibilizar a los ciudadanos sobre problemas locales y globales.

Afinidad de la educación ambiental con el área de trabajo, particularmente ciencias naturales, y el gusto por preservar la vida.

Cambiar las actitudes frente al manejo inapropiado de las instalaciones y los recursos.
Como vemos, las motivaciones más frecuentes de los docentes, para adoptar la educación ambiental a su práctica pedagógica, están relacionadas con la cercanía de sus temáticas a los principios básicos de las ciencias naturales, insistiendo en la transformación de las actitudes y valores en favor de una reconstrucción de las relaciones entre los seres humanos y el medio.

\section{Dificultades en el desarrollo del Prae}

Dentro de las principales dificultades que esbozan los docentes, de manera unánime, en relación con el trabajo ambiental, se hace referencia al tiempo limitado y a la poca flexibilidad curricular de actividades que no hacen parte de la organización habitual del trabajo escolar. Igualmente se reclama una mayor capacitación para la elaboración de los proyectos y la asignación de recursos y espacio para hacer acopio de materiales para los proyectos. En la mayoría de colegios se afirma como dificultad prioritaria la necesidad de recursos económicos para el desarrollo de distintas actividades.

\section{Fortalezas y logros}

Formación de líderes que pueden generar conciencia sobre el ambiente.

El creciente interés de los docentes por participar en los proyectos.

La interdisciplinariedad del proyecto en algunas instituciones.

El apoyo de entidades como Defensa Civil, Departamento Técnico Administrativo del Medio Ambiente, DAMA, Jardín Botánico José Celestino Mutis, entre otras.

Concientizar a la comunidad educativa en cuanto al uso responsable de los residuos sólidos. 
Generación de productos entre los cuales están elementos artísticos a partir de la reutilización y reciclaje de materiales de desecho, la recuperación del espacio público, el embellecimiento de algunos sitios que hacen parte del contexto del colegio, etc.

\section{Proyecciones}

Dentro de las principales proyecciones que visualizan los docentes con respecto al Proyecto Ambiental Escolar se encuentran: la sostenibilidad a futuro de las actividades desarrolladas, el seguimiento a las actividades de reciclaje, el embellecimiento, el incremento en las salidas pedagógicas-ambientales, la creación de vínculos con instituciones de marcada trayectoria en el trabajo ambiental, al igual que el conocimiento de las ofertas ambientales de la localidad. Llama la atención el hecho de dar un peso considerable a las acciones, más que a los procesos de formación de los estudiantes, como parte de las exigencias institucionales.

La tendencia general de las instituciones estudiadas se encamina hacia la simplificación de una realidad, de por sí compleja, propiciando el aislamiento de subsistemas a través de la fragmentación, y olvidando así la interacción y la autoorganización. La necesidad de una mirada interdisciplinaria que permita el aporte de los diferentes saberes a la construcción colectiva del mundo, supone la flexibilización del currículo, fase de la organización institucional, en la que no existe un compromiso claro, por parte de los directivos docentes y los mismos profesores, debido quizás a la dificultad de establecer la toma de decisiones como un aspecto fundamental de procesos de pensamiento complejo, que podrían promover un cambio profundo en la dinámica propia de la escuela y la sociedad.

De hecho, el papel del educador ambiental como dinamizador de la cultura se constituye en uno de los puntos trascendentales que referencian los docentes para la comprensión de la realidad. Como mediadores en la construcción de significados, los docentes estructuran una representación del mundo que involucra las interacciones entre los sujetos y su entorno, convirtiéndose en líderes que integran los conocimientos en favor de la consolidación de una cultura ambiental, conciliando el saber con las necesidades de cada institución y valorando las posibilidades de construcción de una perspectiva ética.

\section{Análisis de las representaciones de ambiente de maestros y estudiantes}

Para la mayoría de los estudiantes y maestros, el ambiente es "lo que nos rodea, es el lugar donde nos encontramos y vivimos, son los seres bióticos, abióticos y el hábitat que estos ocupan”. En este sentido, el ambiente es todo lo que está fuera de nosotros y por esa razón se debe proteger y conservar. Esta situación evidencia la representación naturalista de ambiente que circula en la escuela, debido a la tradición cultural y los desarrollos alcanzados en el abordaje de la ecología para comprender la dinámica de los sistemas naturales. De esta manera no resulta ajeno encontrar en las instituciones educativas que sea el maestro de ciencias y en particular de biología, quien esté a cargo del trabajo ambiental y que a su vez sean los estudiantes quienes 
reconocen que la biología es el área que tiene la mayor relación con las experiencias ambientales de la escuela.

De acuerdo con los respuestas dadas por los maestros $(42 \%)$ y estudiantes (38\%) la representación de ambiente como recurso para administrar se acoge con expresiones como: "El ambiente es un conjunto de recursos que conforman un espacio, del cual el hombre puede sacar provecho o destruir". Esta representación denota la relación que se tiene con el ambiente alejado y escindido del hombre, el cual se asume como un objeto que provee de recursos para satisfacer las necesidades humanas, en cuya base median las acciones que se ejercen sobre éste para acumular riquezas, generar industria, mantener un crecimiento económico que puede ser sostenido, sin que se incorpore una idea de desarrollo sostenible, sin tiempo a futuro y sin compartir con otros grupos humanos.

La representación de ambiente como medio de vida se manifiesta en las respuestas dadas por los docentes (35\%) y los estudiantes (32\%) al ser asumido como "el espacio donde construimos relaciones con otros sujetos y otros seres de la naturaleza; lo bueno y lo malo, todo lo que existe y hace parte del mundo". De esta manera, los problemas ambientales surgen de la relación que establecen los seres humanos con la naturaleza. Estas respuestas sugieren cierta progresión en la comprensión del ambiente, trascendiendo lo biofísico, hacia una conducta relacional que emerge de las interacciones entre los sujetos. A través de los proyectos ambientales se expresa la importancia de contar con un ambiente saludable en el que se establecen unas condiciones adecuadas para el desarrollo de los seres humanos y la conservación de la naturaleza, así como un llamado de atención sobre la forma de habitar el entorno.

La representación de ambiente como problema es asumido en un $22 \%$ por estudiantes quienes expresan que "el ambiente es todo lo relacionado con los ecosistemas, la contaminación por basuras y demás problemas que debemos solucionar" y los maestros, en un $27 \%$ de la muestra, plantean que "es un conjunto de relaciones entre la naturaleza y los seres humanos que pueden afectar el entorno". Si bien se avanza en reconocer que las formas de intervención humana sobre la base natural pueden beneficiar, también reconocen la generación de problemas del orden local, estrechamente articulados con los problemas regionales y globales como se señala en las problemáticas que muestran los estudiantes sobre la contaminación del aire, el agua, los suelos, la destrucción de los ecosistemas, la pérdida de especies y el mal manejo de los residuos sólidos. Este grupo de docentes estima que los problemas ambientales se deben en parte, a la falta de valoración y compromiso con el entorno, además de la falta de conocimiento y capacitación para hacer un mejor manejo de los recursos. Consecuentemente, los maestros reconocen también en los problemas una oportunidad de generar alternativas de solución, de ahí la importancia que éstos le otorgan al trabajo por proyectos en las diferentes instituciones educativas, que tienen como meta educar y transformar las relaciones del ser humano con el entorno.

La representación del ambiente como sistema, de acuerdo con las respuestas 
dadas por los maestros (35\%) y estudiantes (10\%) se evidencia con afirmaciones como: "Es la integración de los seres vivos, los humanos y la naturaleza en un lugar determinado"; "Son las relaciones del hombre, las plantas, los animales y lo inerte". Aquí se muestra que si bien se ha avanzado en entender que existe un fundamento teórico soportado en la teoría de sistemas para interpretar al ambiente como un sistema, hace falta seguir ahondando en esta conceptualización, puesto que en las acciones pedagógicas y didácticas de la escuela es más frecuente trabajar sobre actividades que responden a ciertos propósitos que resulten de fácil concreción y muestre resultados evidenciables. Aunque en ocasiones se trabaje en una acción puntual que puede ser importante, algunos docentes estiman que no resulta articuladora si los estudiantes no encuentran motivación e interés en abordarla, lo que dificulta comprender la red de relaciones que constituye el sistema ambiental.

Sin embargo, vale la pena destacar que este trabajo de discusión y reflexión en torno a la concepción sistémica de ambiente cuenta con un gran interés por parte de maestros y estudiantes, quienes ven en la capacitación para la acción un escenario propicio de implementación de la propuesta.

\section{Análisis de la representación de educación ambiental que manifiestan los estudiantes. Una mirada acerca de los Praey los problemas ambientales}

\section{De la educación ambiental}

El concepto de educación ambiental está en correspondencia con las concepciones de ambiente que los estudiantes tienen: se considera la educación ambiental como las enseñanzas que nos permiten conocer el ambiente para cuidarlo y preservarlo. Existe un grupo de estudiantes que la conciben como un proceso de formación en valores que nos permite construir nuevas relaciones.

\section{Su mirada frente al Prae}

El 75\% de los estudiantes encuestados conocen el Prae de su institución. Usualmente los estudiantes que no pertenecen a los grupos ecológicos o ambientales desconocen el proyecto ambiental de los colegios. La mayoría de estudiantes reconoce el trabajo realizado por los grupos ecológicos, destacando actividades centradas en el reciclaje (38\%), la recolección de residuos sólidos, las campañas de aseo y el ornato (30\%), el arreglo de jardines y la siembra (15\%), las salidas ecológicas (9\%) y el desarrollo académico de proyectos (6\%).

\section{Relación de otras áreas con el Prae}

La mayoría de los estudiantes considera la biología $(86,6 \%)$ y las ciencias sociales (35\%) como las áreas que más contribuyen a conocer los problemas ambientales; se destaca la creciente vinculación de asignaturas como ética, democracia, física y química (26\%); sin embargo, hay áreas que para los estudiantes no tienen relación alguna con lo ambiental, éstas son: matemáticas, educación física y humanidades. Un grupo muy pequeño de estudiantes de algunas instituciones considera que todas las áreas tienen que ver con el ambiente.

\section{Actividades ambientales escolares}

De acuerdo con lo expresado por los estudiantes, las actividades fundamentales del trabajo ambiental para su formación están relacionadas con el cuidado y la preservación de la naturaleza, al igual 
que con los espacios del colegio y la casa donde interactúan. El reciclaje (44\%), las salidas ecológicas (37\%), las actividades de aseo y ornato (28\%), la conformación de grupos ambientales y la participa-ción en talleres lúdico-recreativos (20\%), junto con el desarrollo de proyectos de huerta y lombricultura (14\%), son las actividades ambientales más significativas que reconocen los estudiantes, a la hora de participar activamente. En pocas instituciones se realiza una autoevaluación para identificar las ventajas y desventajas del proyecto y su relación con el proceso de aprendizaje. Consecuentemente, se recalca el conocimiento de los pasos del método científico y su incorporación en el manejo de los residuos sólidos.

La indagación frente al tipo de resultados e intencionalidades de las actividades desarrolladas en el marco de los proyectos ambientales, se centra en la toma de conciencia para ayudar a preservar, ayudar, proteger, respetar o conservar el medio (30\%), lo que demuestra un modelo de educación ambiental de corte naturalista-conservacionista en el que prevalece la modificación de conductas hacia comportamientos proambientales, sin una modificación sustancial del sistema de valores, de los grupos sociales. De esta manera, aprender a cultivar y cuidar las plantas (27\%), aprender técnicas de manejo de residuos sólidos (22\%), conocer y aprender a enfrentar los problemas ambientales (15\%) y embellecer la institución (9\%) permiten concluir que la visión que prevalece en los estudiantes, de acuerdo con las representaciones de educación ambiental que circulan en la escuela, propugna el desarrollo de acciones para enfrentar los problemas concretos, del aquí y el ahora, olvidando las reflexiones y acciones a largo plazo que tienen que ver con transformaciones profundas en nuestro estilo de vida y en los modelos socioeconómicos dominantes.

\section{Reconocimiento de problemas ambientales del orden local, regional y global}

$\mathrm{Al}$ indagar en los estudiantes acerca de cuáles son los principales problemas ambientales, se observa una correspondencia de las respuestas dadas, siendo el mal manejo de residuos sólidos, la contaminación del agua y del aire las que presentan un alto porcentaje de reconocimiento como problema a nivel del colegio, el barrio, la ciudad, el país y el planeta. Esto debido en parte al trabajo intencionado que se desarrolla en las instituciones educativas sobre la situación ambiental actual, así como la información que circula en los diferentes medios de comunicación, que inciden en las formas de concebir dichos problemas (representación globalizante). Quizás también resultan ampliamente reconocidos al ser muy visibles y afectar a la población humana, como es el caso de recursos como el agua y el aire, fundamentales para la existencia y calidad de vida de los seres humanos (representación antropocéntrica).

Otras problemáticas que señalan como importantes corresponden al inadecuado manejo de los recursos naturales, la falta de compromiso y valoración del ambiente, el deterioro de las zonas verdes y la deforestación, lo cual plantea una situación sobre las intervenciones antrópicas, en las que se asume el ambiente como un recurso que provee de bienes y servicios como base de la economía (representación del ambiente 
como recurso), aunque también vale la pena señalar que se preocupan por el sentido que le dan los seres humanos al entorno, haciendo un llamado tácito acerca de la importancia de una moral y una ética más coherente desde unas acciones más armónicas con el ambiente (véase cuadro 1).

Llama la atención que situaciones como la destrucción de la capa de ozono, el calentamiento global y la pérdida de especies, las ubiquen únicamente en la pregunta sobre los problemas ambientales del planeta y no los incluyan en los problemas del país o la ciudad, quizás porque aún exista cierta dificultad en establecer las relaciones y asumir el ambiente como un sistema dinámico y complejo. Así mismo, resulta interesante que un pequeño porcentaje de las respuestas dadas por los estudiantes enunciara el narcotráfico y la violencia como aspectos asociados a la situación ambiental actual del país, lo cual muestra cómo otro tipo de causas pueden estar desencadenando nuevas problemáticas que afectan tanto a la base natural, como a la sociedad y la cultura.

\section{Conclusiones}

Las representaciones sociales sobre ambiente constituyen un modelo de razonamiento común, en el que se revela la diversidad de ideas construidas por los sujetos (docentes y estudiantes) en interacción con el grupo social en el que conviven; prueba de ello es la aceptación

\begin{tabular}{|c|c|c|c|c|c|}
\hline $\begin{array}{ll} & \text { Contexto } \\
\end{array}$ & Colegio & Barrio & Ciudad & País & Planeta \\
\hline Mal manejo de residuos sólidos & $58,3 \%$ & $73,3 \%$ & $60 \%$ & $66,6 \%$ & $73,3 \%$ \\
\hline Contaminación del agua & & $15 \%$ & $40 \%$ & $60 \%$ & $71,6 \%$ \\
\hline Desperdicio del agua & $13,3 \%$ & & & & \\
\hline Deterioro de zonas verdes & $38,3 \%$ & $48,3 \%$ & $13,3 \%$ & & \\
\hline Contaminación auditiva & $15 \%$ & $11,6 \%$ & $16,6 \%$ & $30 \%$ & $45 \%$ \\
\hline Falta de compromiso y valoración del ambiente & $8,3 \%$ & $48,3 \%$ & $6,6 \%$ & & $28,3 \%$ \\
\hline Contaminación del aire & & $25 \%$ & $56,6 \%$ & $43,3 \%$ & $63,3 \%$ \\
\hline Contaminación visual & & $10 \%$ & $11,6 \%$ & $21,6 \%$ & $21,6 \%$ \\
\hline Contaminación del suelo & & $11,6 \%$ & & & \\
\hline Deforestación & & & $13,3 \%$ & $58,3 \%$ & $33,3 \%$ \\
\hline Falta de zonas verdes & & $13,3 \%$ & & $3,3 \%$ & \\
\hline Mal manejo de los recursos naturales & & & & $58,3 \%$ & \\
\hline Narcotráfico & & & & $5 \%$ & \\
\hline Violencia que genera contaminación & $3,3 \%$ & & & $6,6 \%$ & \\
\hline Destrucción de la capa de ozono & & & & & $20 \%$ \\
\hline Extinción de especies & & & & & $10 \%$ \\
\hline Calentamiento global & & & & & $5 \%$ \\
\hline Desconocimiento del funcionamiento de la naturaleza & $10 \%$ & & & & \\
\hline
\end{tabular}

Cuadro 1. Reconocimiento de problemas ambientales del orden local, regional y global por parte de los estudiantes. 
de ciertos esquemas espontáneos de razonamiento, en los que prevalece una clara alusión al conocimiento local como estrategia de pensamiento habitual, confirmándose la presencia de representaciones de ambiente que propugnan la preservación, la conservación, el respeto y el cuidado, de acuerdo con las ideas de corte ambientalista que hacen parte de las vivencias de los sujetos, las cuales circulan en el contexto familiar y son promovidas por los medios de comunicación y la escuela (representación de ambiente como naturaleza).

En nuestra sociedad, las representaciones sociales sobre el ambiente se caracterizan por la escisión entre naturaleza y cultura (Noguera, 2004), lo cual confluye en expresiones de dominio que hoy se mantienen, en una clara apología del análisis, la fragmentación y el conocimiento reticular (representación naturalista). De hecho, para la mayoría de docentes y estudiantes se consideran como problemas a abordar desde la educación ambiental, aquellos que exclusivamente se centran en la conservación y preservación de espacios verdes, confirmando la separación entre subsistemas biofísicos y socioculturales. En términos pedagógicos y didácticos, esta división se apoya en los proyectos ambientales que reviven ciertos activismos con los que se pretende trasladar en espacios reducidos, el contexto rural a la ciudad (arborización, disminución de residuos sólidos, agricultura urbana, salidas, etc.). Algunas de las propuestas manifiestan una visión de la sociedad como oposición entre lo rural y lo urbano, estableciendo como objetivo estratégico la búsqueda de espacios relativos que distinguen lugares dentro del conjunto de la ciudad, en los cuales se encuentran anclados vestigios de una identidad ambiental (representación de ambiente como recurso para administrar).

Si bien es cierto que se ha progresado en relación con aquella representación antropocéntrica, en la cual el hombre maneja los recursos para su sobrevivencia, existen serias limitaciones a la hora de comprender que somos parte de la naturaleza. Así, la aceptación por parte de la mayoría de docentes y estudiantes de representaciones de ambiente de corte naturalista, reafirma la robusta tradición del uso del medio, propugnada por pedagogos como Freinet (citado por Hagoury, 2000), en la que se contempla la enseñanza como el desarrollo de habilidades tanto observacionales como experienciales, en aquel lugar con recursos que se deben conservar y preservar. En este sentido, se entrelazan las ideas de maestros y estudiantes al asumir que el ambiente en su base natural provee de recursos a los seres humanos para satisfacer sus necesidades, pero que cuando es sobreexplotada presenta serios problemas del orden local, regional y global y, por tanto, se requiere de una educación que promueva su conservación, la cual se logra con el desarrollo de proyectos ambientales escolares (representación de ambiente como problema por resolver).

Vale la pena mencionar que para un número creciente de docentes, un aspecto clave en el estudio de los sistemas lo constituye el establecimiento de las propiedades emergentes, es decir aquellas cualidades que surgen en cierto nivel de complejidad pero que no se presentan en niveles inferiores. Para el caso particular de la educación ambien- 
tal, se entiende que las propiedades emergentes no están contenidas en las partes del sistema, surgen de la misma organización del conjunto, retroactuando sobre él (re-presentación de ambiente como biosfera). Se poodría afirmar que la concientización tan promocionada en los proyectos ambientales, es una emergencia del subsistema social que se convierte en un nuevo elemento que nace de la organización del conjunto. Pero hablar de concientización implica ir más allá de entender las características de los problemas y sus consecuencias, significa sensibilizar hacia los conflictos, en particular los de interés, reconociendo las razones políticas que los orientan (representación globalizante).

Coincidimos con la tesis de algunos teóricos de la educación ambiental, como Caride y Meira (2001), quienes señalan que muchas de estas propuestas, en las que se utilizan los términos concientización y sensibilización se corresponden con un paradigma mecanicista por cuan-

\section{Bibliografía}

Caride, J. A y Meira, P. A. (2001). Educación ambiental y desarrollo humano. Barcelona: Editorial Ariel.

Cerda, H. (1998). Los elementos de la investigación. Bogotá: Editorial El Búho.

Decreto 1743 de 1994. Diario Oficial N. ${ }^{o}$ 41476, 5 de agosto de 1994.

García, J. E. (2002). Los problemas de la educación ambiental: ¿Es posible una educación ambiental integradora? En: Nuevas Tendencias de la Educación Ambiental. Colección Investigación en la Escuela, N. ${ }^{\circ} 46$. Sevilla: Díada Ediciones. to las personas se concentran en conocer una realidad física, objetiva y dada, desconociéndose diversas interpretaciones e interacciones que involucran el estudio de los problemas ambientales dentro de una perspectiva política y socialmente conflictiva (representación de ambiente comunitario). De hecho, muy pocos docentes advierten sobre la relación entre el creciente deterioro ecológico y las consecuencias sociales de la movilización de la población más vulnerable de nuestro país. Bogotá como ciudad capital recibe constantemente familias de desplazados, hecho que contribuye a una modificación estructural de la sociedad, convirtiéndose en destino de quienes han perdido parte de su identidad frente al entorno y la cultura de origen, siendo esta una clara manifestación de aislamiento y discriminación frente a intereses económicos o de poder que ven como patrimonio el deslegitimar la multiplicidad de discursos y argumentaciones. $\boldsymbol{\Delta}$

Hagoury, R. (2000). El arte de la indagación en el aula. Barcelona: Gedisa.

Jodelet, D. (1986). La representación social. Conceptos y teoría. En: Moscovici, S. Psicología social, Vol. II. Pensamiento y vida social. Psicología social y problemas sociales. Madrid: Paidós.

Moscovici, S. (1979). El psicoanálisis, su imagen y su público. Buenos Aires: Huemal.

Noguera, P. (2004). Reencantamiento del mundo. Manizales: Universidad Nacional de Colombia, Instituto de Estudios Ambientales, IDEA. 
Novo, M. (2003). La educación ambiental. Bases éticas, conceptuales y metodológicas. Madrid: Editorial Universitas.

Pérez, R. y Porras, Y. (2005). La complejidad en el marco de una propuesta pluriparadigmática. Tecné Episteme y Didaxis, 17. Bogotá: Universidad Pedagógica Nacional.

Pérez, R. et. al. (2004). Un estudio para identificar tendencias en ambiente $y$ educación ambiental en instituciones educativas del Distrito Capital. Informe final. Bogotá: Centro de Investigaciones de la Universidad Pedagógica Nacional.

Pozo, J. I. y Gómez, M. A. (1998). Aprender y enseñar ciencias. Del conocimiento cotidiano al conocimiento científico. Madrid: Ediciones Morata.

Reigota, M. (1995). Meio ambiente e representaçoes sociais. Sao Paulo: Editora Cortez.
Sauve, L. (1994). Dimensión ambiental y la escuela. Seminario Internacional. Bogotá: Ministerio de Educación Nacional.

Talero, E. y Umaña, G. (1998). Aportes para la realización del proyecto de educación ambiental. Bogotá: Ediciones Romero.

Sanmartí, N. y Pujol, R. M. (2002). ¿Qué comporta capacitar para la acción en el marco de la escuela? En: Nuevas Tendencias de la Educación Ambiental. Colección Investigación en la Escuela, N. ${ }^{\circ} 46,49-54$. Sevilla: Diada Ediciones.

Torres, M. (1996). La dimensión ambiental: un reto para la educación de la nueva sociedad. Bogotá: Ministerio de Educación Nacional.

Varela, F. (1996). Ética y acción. Santiago de Chile: Dolmen Editores. 